\title{
EXPERIENCES WITH COMMISSIONING SOFTWARE TOOLS AT BESSY II *
}

\author{
R. Bakker, T. Birke, B. Kuske, R. Lange, R. Müller ${ }^{\dagger}$, BESSY, Berlin, Germany
}

\section{Abstract}

Software requirements evolve rapidly during the short commissioning period of a third generation light source. Main focus moves from atomic device control to complex conditioning, experiment performance, measurement data taking, beam quality maintenance and performance monitoring tasks. From the very beginning the essential tools have to be set up properly and need to be sufficiently adaptive to easily absorb the actual findings of the running in procedures. In this paper the major software modules at BESSY II are depicted and their relevance for the progress of the commissioning is rated. Implemented functionalities and used methods are described in more detail for the basic working horses (like compound devices, handling of snapshot files, orbit control). Explanations are given why certain tools turned out to be less accepted or useful (yet).

\section{APPLICATION DEVELOPMENT ENVIRONMENT}

With respect to software requirements commissioning of a light source storage ring is a very demanding phase. Tools with a wide variety of functionalities have to be available (see fig. 1). Many new insights into the behaviour of the new machine are achieved in a very short time. Numerous requests for creation or modification of software support arise.

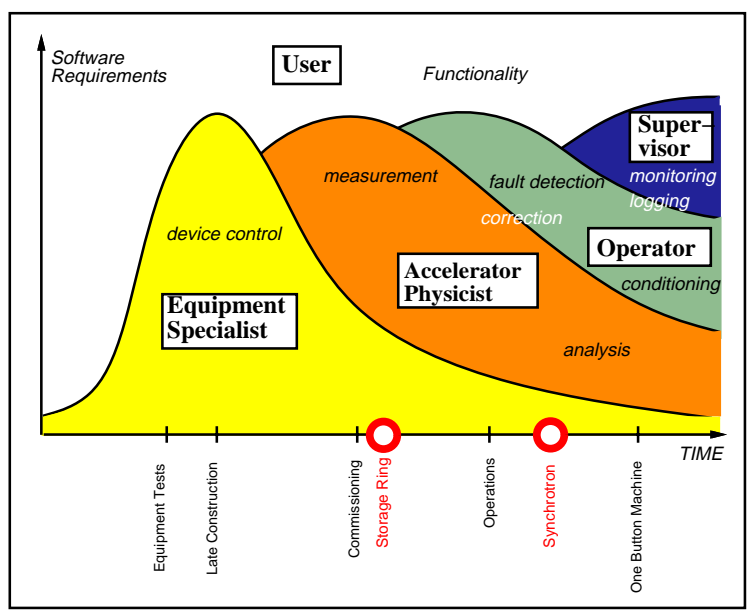

Figure 1: Typical Lifecycles of Operations Software

Based on the EPICS toolkit used at BESSY a large number of generic applications is available that noticably facil-

\footnotetext{
* Funded by the Bundesministerium für Bildung, Wissenschaft Forschung und Technologie and by the Land Berlin

† Email: mueller@bii.bessy.de
}

itates the set up of a device control layer. In addition a few tools are suited to satisfy operator or supervisor requests. Standard measurement and analysis tasks are feasible with the aid of the SDDS toolkit[1] and shell programming.

For performance analysis and correction procedures involving accelerator physics describing accelerator behaviour a number of solutions and toolkits developed in other laboratories had been investigated. Amount of adaptation work, resulting flexibility and related arguments led to the decision to pick up ideas and create these programs within the software context available at BESSY. For that purpose three fundamental design decisions turned out to be very helpful: to use (1) the relational DB ORACLE as global reference repository, (2) the unique $\mathrm{C}++$ toolkit goemon[2] for model descriptions, (3) a well separated interface to the graphical user interface[3]. That infrastructure allowed to build additional utility toolkits e.g. a magnet-power supply support library convenietly connecting physics and model description with device I/O and engineering units.

\section{DEVICE CONTROL}

\subsection{Status Displays, Control Handles}

Comprehensive synoptic views have been built with the EPICS display manager that allow for hierarchical structured navigation and provide a web of light weight and user friendly control panels. For specific tuning problems a knob manager connects the setpoints of interest to dial boxes. Strip chart recording software is available for simple stability analysis as well as for correlation tests and preliminary measurements. Supervisory tasks take care of sensitive devices, e.g. sending OFF to the microtron contrapole causes a software controlled graceful ramp down procedure. Thus for the control of single devices a intuitive, 'complete', and robust man-machine interface has been set up that is well accepted and adequately operatable from a single operator seat equipped with two monitors and five workspaces.

\subsection{Pseudo Devices}

Dependent on the operation mode quadrupole and sextupole power supplies are combined to families. Or the kicker amplitudes have to be tuned until a closed bump is formed. Then the closed bump amplitude has to be optimized for injection efficiency. To operate these groups of devices by pseudo set points has been made possible through sophisticated programming on the front end computer (IOC) level. Especially in the early phase of beam threading the rapid, easy and consistent switching between single device access and groupwise operation turned out to be extremely helpful. 
For temporary, not yet settled or less elementary applications an easy configurable 'multi-knob' is available on the work station level. It is e.g. used for the energy scaling of transfer line and storage ring or the orthogonal adjustment of vertical or horizontal tune and chromaticity.

\section{MACHINE CONDITIONING}

\subsection{Save / Restore}

For literally every setpoint there is a global and persistent memory field available. This is very convenient for manual tuning procedures. With mouse clicks one can save the starting point or the local optimum and return to this point when the attempt to improve does not promise success.

For global storing and reloading of relevant machine settings a tcl/Tk program overcomes the typical problems connected with the classical approach to dump all data into one file. The script provides a very transparent framework for handling snapshot file catalogs, data aging and partial recall. By a flexible and hierarchical area selection mechanism arbitrary collection of devices can be quickly defined in both dimensions: equipment location and device type. The corresponding setpoints (and readbacks) can be stored in file sets, that are completely hidden from the users. Reference is done by arbitrary comments, date and time or serial number. Partial or subsequent re- or overloading is feasible by definition of sub-areas or by specification of filter functions.

For a specified device collection it is possible to compare (in any combination) setpoint and readback values of snapshot files and corresponding values actually in effect for the running machine. Differences exceeding a configurable percentage are highlighted. For administration purposes the user may remove files (into the attic) or add it to the list of favourite files. For offline analysis the export options 'print out', 'dump to file' or 'send email' are widely used. By its structure this facility is easy maintainable and adaptable. It is well accepted, robust and very reliable.

\subsection{Cycling Procedures}

Only specific devices (e.g. microtron linac) are conditioned by programs running on the IOC. For procedures involving groups of devices the adaptation of an appropriate tool [4] has been foreseen but had to be postponed. As an interim solution a tcl/Tk script has been set up for the cycling of the storage ring and transfer line magnets. Main functionality of the script is to call the database, group the power supplies, set up and run the sequence. This quick solution encountered problems trying to meet unclear given specifications and to absorb rapidly changing requirements. The initially insufficient operational safety has been quickly overcome, but for future operations it will be replaced and embedded into a more general sequencer facility.

\subsection{Alarms}

As soon as new devices have been stable and continously available they have been included into the alarm handler configurations. Major or minor error conditions for the areas microtron, transfer lines, booster, storage ring, RF system and magnet interlock are reported. In a structured and hierarchical way the operator is guided to the malfunctioning device and provided with the appropriate control panel needed to analyse and fix the problem. For fault detection it increasingly replaces the use of synoptic views. For further analysis received alarms are logged to files.

\section{DATA AQUISITION AND EVALUATION}

\subsection{Measurements, Analysis}

During commissioning systematic measurements are a difficult field of improvisation. Both machine, control system and software tools are not yet familiar. Mostly coarse manual scans dominate. Further evaluation of data is done with PC standard office tools or dedicated programs that are usable only by the author. Occasionally the correlation engine Xtract [5] has been used for scraper measurements, one and two-dimensional tune scans or stepping of the harmonic sextupoles to find the best dynamic aperture.

Only orbit correlations are taken systematically and a couple of standardized analysis programs are available. Routinely corrector response matrices are measured and postprocessed offline (e.g. [6]). In the beam based alignment set-up an additional power supply is switched to all quadrupoles and stepped automatically and the response matrices are used to determine the BPM offsets[7].

\subsection{Data Logging}

With respect to the importance of an archive containing all signals suited to identify unknown sources of performance degradation the present situation is a minimum effort solution. Beam current, lifetime, orbit, vacuum, power supply stability data are sampled and stored in binary format. Only for orbit data and correlated signals a display, retrieval and correlation browser exists that is configurable with a graphical user interface. Beam intensity and vacuum data are converted to standardized graphics accessible by WEB navigators. Shortcoming of the SDDS based data collectors are the fixed time intervals between data aquisition. Frequently notification on data change is appropriate. The continuously increasing demand on archived signals emphasizes the embryonic state of the present system as well as the need for a major development effort.

\section{ACCELERATOR PHYSICS}

\subsection{Orbit}

Data aquisition, display and control of the orbit in all segments of the accelerator complex, i.e. transfer lines, synchrotron (SY) and storage ring (SR) is handled by a generic, 
event driven program called Orbit. It features a 'All-inOne' graphical user interface consisting of a main program control panel, a context sensitive BPM data display and a context sensitive corrector control panel.

The main panel is for display of statistical summary, setup of the fundamental orbit control types, selection of basic measurement modes, configuration of data I/O, modification of BPM system operation parameters and display of program informations, warnings and error reports.

BPM data are displayed as high precision averaged closed orbit, specific turn number of single turn data (SR), orbit at a specific energy during the ramping cycle (SY) - both absolute and as difference to an arbitrary reference orbit. For each individual BPM stability of displacement, intensity, averaging quality is recorded and statistically analysed. Additionally turn-by-turn changes (SR) or energy dependencies (SY) at a selectable BPM can be displayed. BPM data may be logged to files together with arbitrary correlation channels.

One fundamental orbit control mode are closed bumps. Required bumps may be choosen from a list (e.g. center of insertion device, achromat) or freely configurable with arbitrary target position and corrector combination. According to requested amplitude and angle bumps are calculated with the embedded online-model according to the actual set points of the accelerator. The bump predictions are reliable on the percent level. A systematic bump scan during start up of the storage ring helped to pinpoint destructive aperture limitations within one meter[8].

Global orbit correction to an arbitrary reference orbit is the other control mode. Standard correction method is based on Singular Value Decomposition (SVD). For fault analysis the most effective corrector method is available too. Calculated model values or experimental results supply response matrices. On faulty BPM reading or corrector status change the elements are automatically removed from the correction scheme until they are available again. The operator may manually exclude or reenable BPMs or correctors with a mouse click. The reference orbit may be choosen from beam based alignment data (optimized) and the logging archive (comparison). It can be modified with mouse drags (bumps).

Two automatic correction schemes are presently under study: (1) a mixture of fixed frequency (e.g. $0.1 \mathrm{~Hz}$ ) and a deviation threshold (e.g. two times the noise) (2) a PID type regulator. Both methods converge relatively fast and keep the RMS orbit deviations stable at the optimal value but the minimal invasive method with optimal reduction of residual noise is not yet identified.

The program is an accurate working horse. Its basic functionality has been set up and consolidated for the booster. As a consequence it worked reliable at start-up of the storage ring. Without modification the embedded online model allowed to correct the orbit at all presently established energies $(0.9,1.7$ and $1.9[\mathrm{GeV}])$ with comparable precision.

\subsection{Optics}

A generic online model program called Optics helps to understand certain tunings of any segment of the accelerator complex (transfer lines, SY, SR). Interface to the real accelerator is the capability to load and save snapshot files and to read and modify power supply setpoints. The program displays beta function, trajectory and dispersion. It calculates the spot size and position on an intercepting fluorescent screen. To allow studies of dependencies on parameter variation input controllers for power supply (family) setpoints, beamline matching conditions and extraction / injection bump parameters are available. The influence of fundamental model approximations can be compared: standard hard edge magnets, current dependant conversion factors or saturation induced variable magnetic length. For the rings fitting procedures for requested fractional tune or chromaticity are available. Tune adjusting quadrupoles can be restricted to selectable families.

The program is frequently very useful. Precision is approaching reality. Since there is no online data source for tune and chromaticity available yet and there has not been a commitment for a serious and systematic testing so far Optics has still the quality of a beta release.

\section{EXPERIENCES, SUMMARY}

The mature EPICS toolkit provided the framework for a powerful basic operational set. Within the BESSY software context generic applications have been built that evolved 'downsteam' with the installation process. For the commissioning an adequate set of easy to use and reliable operations program has been available. The software system is transparent and robust. It could mostly adopt requests for changes without noticible delays or inconsistencies. Frequently new functionalities have been anticipated and installed when the existing features began to be appreciated. A number of additional tools are ready to be set up for various tasks. They lie idle because utilization would require a certain user programming capability and effort.

\section{REFERENCES}

[1] M. Borland, L. Emery, N. Sereno, Proceedings of the 1995 ICALEPCS, Chicago, 1995, p.382

[2] H. Nishimura, Nucl. Instr. Meth., A 352 (1994), p.379

[3] R. Bakker et al., Proceedings of the 1998 EPAC, Stockholm, 1998, p.1676

[4] T. Birke, R. Lange, R. Müller, Proceedings of the 1995 ICALEPCS, Chicago, 1995, p.648

[5] Xtract has been developed at TJNAF. See: http://help.bessy.de/xtract/xtract.html.

[6] B. Kuske et al., 'Establishment of a Model for Interpretation and Correction Tools for BESSY II', this conf. (WEP48).

[7] P. Kuske et al., 'Experience with the BPM System of the BESSY II Storage Ring', this conference (WEA84).

[8] R. Bakker et al., 'Status and Commissioning Results of BESSY II', this conference (TUCL3). 